\title{
First principles calculations of optical properties for oxygen vacancies in binary metal oxides
}

Cite as: J. Chem. Phys. 150, 044702 (2019); https://doi.org/10.1063/1.5078682

Submitted: 27 October 2018 . Accepted: 02 January 2019. Published Online: 28 January 2019

Jack Strand (D), Sergey K. Chulkov (D), Matthew B. Watkins (D), and Alexander L. Shluger (iD)
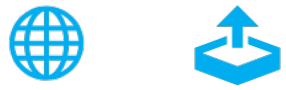

\section{ARTICLES YOU MAY BE INTERESTED IN}

The surface affinity of cations depends on both the cations and the nature of the surface The Journal of Chemical Physics 150, 044706 (2019); https://doi.org/10.1063/1.5065075

Simulated XUV photoelectron spectra of THz-pumped liquid water

The Journal of Chemical Physics 150, 044505 (2019); https://doi.org/10.1063/1.5054272

Theoretical analysis of screened many-body electrostatic interactions between charged polarizable particles

The Journal of Chemical Physics 150, 044901 (2019); https://doi.org/10.1063/1.5079515 


\title{
First principles calculations of optical properties for oxygen vacancies in binary metal oxides
}

\author{
Cite as: J. Chem. Phys. 150, 044702 (2019); doi: 10.1063/1.5078682 \\ Submitted: 27 October 2018 - Accepted: 2 January 2019 • \\ Published Online: 28 January 2019
}

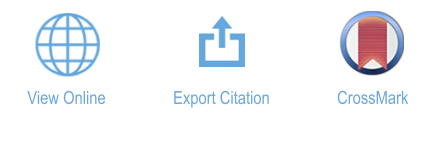

Jack Strand, ${ }^{1, a)}$ (D) Sergey K. Chulkov, 2,b) (D) Matthew B. Watkins, ${ }^{2, c)}\left(\mathbb{D}\right.$ and Alexander L. Shluger, ${ }^{3, d)}$ (D)

\begin{abstract}
AFFILIATIONS
${ }^{1}$ Department of Physics and Astronomy, University College London, Gower Street, London WC1E 6BT, United Kingdom

${ }^{2}$ School of Mathematics and Physics, University of Lincoln, Brayford Pool, Lincoln LN6 7TS, United Kingdom

${ }^{3}$ Department of Physics and Astronomy and London Centre for Nanotechnology, University College London, Gower Street, London WC1E 6BT, United Kingdom
\end{abstract}

a) Electronic mail: jack.strand.74@ucl.ac.uk

b) Electronic mail: schulkov@lincoln.ac.uk

${ }^{c}$ Electronic mail: mwatkins@lincoln.ac.uk

d) Electronic mail: a.shluger@ucl.ac.uk

\begin{abstract}
Using an advanced computational methodology implemented in CP2K, a non-local PBE0-TC-LRC density functional and the recently implemented linear response formulation of the Time-dependent Density Functional Theory equations, we test the interpretation of the optical absorption and photoluminescence signatures attributed by previous experimental and theoretical studies to O-vacancies in two widely used oxides-cubic $\mathrm{MgO}$ and monoclinic $(\mathrm{m})-\mathrm{HfO}_{2}$. The results obtained in large periodic cells including up to 1000 atoms emphasize the importance of accurate predictions of defect-induced lattice distortions. They confirm that optical transitions of $\mathrm{O}$-vacancies in $0,+1$, and +2 charge states in $\mathrm{MgO}$ all have energies close to $5 \mathrm{eV}$. We test the models of photoluminescence of $\mathrm{O}$-vacancies proposed in the literature. The photoluminescence of $\mathrm{V}_{\mathrm{O}}^{+2}$ centers in $\mathrm{m}-\mathrm{HfO}_{2}$ is predicted to peak at $3.7 \mathrm{eV}$ and originate from radiative tunneling transition between a $\mathrm{V}_{\mathrm{O}}^{+1}$ center and a self-trapped hole created by the $5.2 \mathrm{eV}$ excitation.
\end{abstract}

Published under license by AIP Publishing. https://doi.org/1 0.1063/1.5078682

\section{INTRODUCTION}

Oxygen vacancies strongly affect physical and chemical properties of oxides and have been studied extensively both experimentally and theoretically. ${ }^{1-4}$ Experimental identification of such defects in bulk materials often relies on the interpretation of recorded optical absorption, photo-luminescence, and electron paramagnetic resonance spectra. Surprisingly, spectroscopic signatures of $\mathrm{O}$ vacancies in different charge states are not well established even in case of the simplest oxides, such as MgO. This complicates the verification of existence of $\mathrm{O}$ vacancies as well as models and hypothesis attributed to effects of their presence. In this paper, we test the interpretation of optical absorption and photoluminescence (PL) signatures attributed in the literature to $\mathrm{O}$ vacancies in two widely used metal oxides-cubic $\mathrm{MgO}$ and monoclinic (m) $-\mathrm{HfO}_{2}$.

$\mathrm{MgO}$ has long been studied as a model wide bandgap oxide with numerous technological applications. Extensive experimental studies have been carried out of defects in $\mathrm{MgO}$ and particularly O-vacancy (also known as an F center). ${ }^{5-10}$ Theoretical studies, however, still struggle to give reliable insights into the optical spectra of different charge states of this defect. For example, theoretical models still continue to disagree on the exact positions of optical absorption peaks of neutral and positively charged vacancies. The first optical absorption peaks of the neutral and +1 charged oxygen vacancies $\left(\mathrm{V}_{\mathrm{O}}^{0}\right.$ and $\mathrm{V}_{\mathrm{O}}^{+1}$ centers) occur at very similar energies: 5.01 and $4.96 \mathrm{eV}$, respectively. ${ }^{5}$ These peaks are difficult to resolve both experimentally and in theoretical calculations. Ab initio cluster model calculations of $\mathrm{V}_{\mathrm{O}}^{0}$ and $\mathrm{V}_{\mathrm{O}}^{+1}$ defects ${ }^{11}$ predicted 
very similar optical absorption energies; however, the absolute value was closer to $6 \mathrm{eV}$, rather than the experimental $5 \mathrm{eV}$. It was argued that the main source of error in this study was the limited basis set size and that $3 d$ polarization functions should be added to the oxygen basis set. Further calculations ${ }^{12}$ using a larger basis set predicted the optical absorption energy for $\mathrm{V}_{\mathrm{O}}^{0}$ at $5.44 \mathrm{eV}$, in closer agreement with the experiment. The optical excitation energy for the $\mathrm{V}_{\mathrm{O}}^{+1}$ defect, however, was not improved. It was therefore concluded that much larger basis sets are necessary for the accurate prediction of the optical absorption spectrum. In both of these studies, only excitations in the alpha spin channel were reported for the $\mathrm{V}_{\mathrm{O}}^{+1}$ center. However, there are, in fact, two types of optical excitation in the $\mathrm{V}_{\mathrm{O}}^{+1}$ center (see Fig. 1): a type- $\mathrm{V}$ transition into a higher state located close to the bottom of the conduction band (CB) in the $\alpha$-spin channel and a type-III transition from the defect-induced valence band (VB) states into the unoccupied state associated with the $\mathrm{V}_{\mathrm{O}}^{+1}$ center in the $\beta$-spin channel.

The $\beta$ channel excitation was suggested in previous theoretical and experimental studies ${ }^{13,14}$ as a possible origin of the $5 \mathrm{eV}$ absorption peak. Interestingly, both the $\alpha$ and $\beta$ channel excitations (type $\mathrm{V}$ and type III, correspondingly) in the $\mathrm{V}_{\mathrm{O}}^{+1}$ center are predicted to be close to $5 \mathrm{eV}$. Thus, all three optical absorption peaks associated with $\mathrm{V}_{\mathrm{O}}^{0}$ and $\mathrm{V}_{\mathrm{O}}^{+1}$ centers have very close energies. A qualitative difference between $\alpha$ and $\beta$ spin excitation for the $\mathrm{V}_{\mathrm{O}}^{+1}$ center, however, is that $\beta$ channel excitations should be associated with the release of holes into the valence band (VB). It has been shown that, when exciting $\mathrm{MgO}$ crystals with $5 \mathrm{eV}$ light, a $2.3 \mathrm{eV}$ absorption band also develops, which is associated with holes trapped on $\mathrm{V}-$ type centers. ${ }^{13,14}$ Recent periodic Density Functional Theory (DFT) calculations ${ }^{15}$ account for different spin channel excitations in $\mathrm{MgO}$ using many-body perturbation theory in the $\mathrm{G}_{0} \mathrm{~W}_{0}$ approximation and the Bethe-Salpeter approach. These

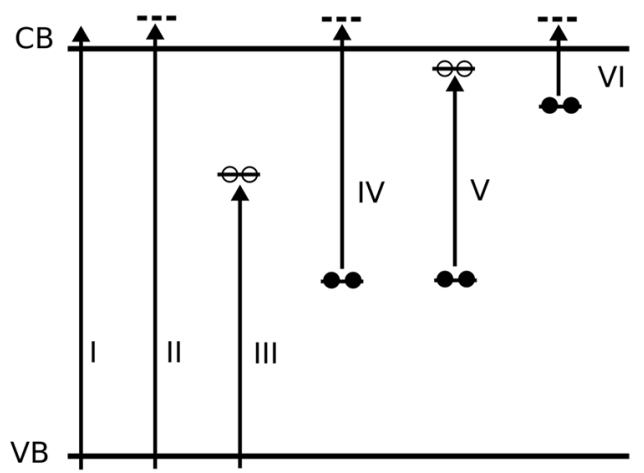

FIG. 1. Schematic of the typical optical transitions in oxides with defects. TypeI is a band-to-band transition. Type II is a VB to the defect-induced unoccupied resonant state in CB transition. Type III represents the VB to the unoccupied defect state in the bandgap transition. Type IV is an occupied defect state into defectinduced state in the CB transition, and type $V$ is an occupied defect state into un-occupied defect state in the bandgap transition. A type VI transition is from a shallow, occupied defect state into the defect-induced resonant state in the CB. calculations predicted the existence of a $3.6 \mathrm{eV}$ absorption band in the $\mathrm{V}_{\mathrm{O}}^{+1}$ center (in addition to the known $5 \mathrm{eV}$ band in the $\alpha$ channel), which could be used to distinguish between the $\mathrm{V}_{\mathrm{O}}^{0}$ and $\mathrm{V}_{\mathrm{O}}^{+1}$ centers. Thus the position and nature of optical absorption peaks of $\mathrm{V}_{\mathrm{O}}^{0}$ and $\mathrm{V}_{\mathrm{O}}^{+1}$ centers in $\mathrm{MgO}$ remain controversial. They are revisited and discussed in more detail in Sec. III B.

$\mathrm{MgO}$ also has a photoluminescence (PL) band at $2.3 \mathrm{eV}$ attributed to the $\mathrm{V}_{\mathrm{O}}^{0}$ center. $^{8-10,16}$ The nature of this luminescence has been discussed based on theoretical and experimental data in Refs. 16 and 17 but has not been confirmed by many-electron calculations. These models are tested and discussed in detail in Sec. III C.

$\mathrm{HfO}_{2}$ is another topical oxide with many properties attributed to $\mathrm{O}$ vacancies. This high dielectric permittivity oxide is being used both as a gate dielectric in modern transistors and as a reducible oxide in memory cells. ${ }^{18,19}$ The existing and newly generated oxygen vacancies in $\mathrm{HfO}_{2}$ have been implicated in degradation of these devices and play the central role in the electroforming process in Resistive Random Access Memory (RRAM) devices. ${ }^{20-23}$ Again, spectroscopic signatures of these defects are still controversial.

Experimentally, there is a well characterized $2.7 \mathrm{eV}$ photoluminescence peak ${ }^{24,25}$ associated with a $5.2 \mathrm{eV}$ absorption peak. The optical transitions for all five charge states of the oxygen vacancy in $\mathrm{m}-\mathrm{HfO}_{2}$ were calculated in a DFT study using both periodic and embedded cluster methods and Time-dependent DFT (TD-DFT) for calculating optical transition energies. ${ }^{26}$ These calculations predicted the absorption energy of the +2 charged oxygen vacancy $\left(\mathrm{V}_{\mathrm{O}}^{+2}\right.$ defect) at $4.94 \mathrm{eV}$, which is appreciably close to the $5.2 \mathrm{eV}$ absorption peak. This provided evidence that the $\mathrm{V}_{\mathrm{O}}^{+2}$ defect may be responsible for the $5.2 / 2.7 \mathrm{eV}$ absorption/PL spectrum. Other experimental work on hafnia films has also connected this luminescence peak to oxygen vacancies. In a photoluminescence study, ${ }^{25}$ the $5.2 \mathrm{eV}$ absorption and the $2.7 \mathrm{eV}$ luminescence bands were interpreted as emerging from neutral (rather than positively charged) oxygen vacancies. Furthermore, another absorption/emission line-a $3.66 \mathrm{eV}$ PL excited at $4.4 \mathrm{eV}$ and $5.4 \mathrm{eV}$-has also been detected. ${ }^{27}$ The predicted optical absorption energy for the neutral vacancy is approximately $3.2 \mathrm{eV} .^{26}$ This corresponds to an electron being excited out of a doubly occupied vacancy state [which has its KohnSham (KS) level in the middle of the bandgap] into an unoccupied state at the bottom of $\mathrm{CB}$ (type $\mathrm{V}$ excitation in Fig. 1). These calculations also predict similar positions for absorption peaks of $\mathrm{V}_{\mathrm{O}}^{-1}, \mathrm{~V}_{\mathrm{O}}^{0}$, and $\mathrm{V}_{\mathrm{O}}^{+1}$ defects, which all have occupied states at similar energies in the bandgap.

Thus, rather surprisingly considering the amount of research which went into studies of these two materials, their optical absorption and photoluminescence (PL) spectra are far from being understood. Predictions of positions of defect optical absorption and PL peaks rely on several factors: accuracy of calculations of defect-induced lattice relaxation, which often requires large periodic cells or clusters; positions of defect levels should be accurately reproduced, which requires using non-local density functionals; and techniques used to calculate optical excitation energies. 
Satisfying all these requirements is challenging, and compromises are often made in favor of one of the variables. For example, small periodic cells are used to afford more computationally demanding calculations using the GW approximation and the Bethe-Salpeter approach for calculating optical spectra. More efficient but less accurate local density approximation (LDA) calculations are used to optimize the defect geometry.

Here we attempt to satisfy all the requirements by using an advanced computational methodology implemented in $\mathrm{CP} 2 \mathrm{~K}$, a non-local PBE0-TC-LRC density functional and the recently implemented linear response (LR) formulation of the TD-DFT equations. These are described in detail in Sec. II and in the Appendix. Using this method, within the adiabatic approximation, we calculate the optical transition energies for the $0,+1$, and +2 charged $\mathrm{O}$ vacancies in $\mathrm{MgO}$ and $\mathrm{m}-\mathrm{HfO}_{2}$ and estimate the photoluminescence energies for the $\mathrm{V}_{\mathrm{O}}^{0}$ and $\mathrm{V}_{\mathrm{O}}^{+2}$ centers in $\mathrm{MgO}$ and $\mathrm{m}-\mathrm{HfO}_{2}$. The results emphasize the importance of accurate predictions of defect-induced lattice distortions. They confirm that optical transitions of $\mathrm{O}$-vacancies in $0,+1$, and +2 charge states in $\mathrm{MgO}$ all have energies close to $5 \mathrm{eV}$. We qualitatively confirm the model proposed to explain the nature of $2.3 \mathrm{eV} \mathrm{PL}$ in $\mathrm{MgO}$ in Refs. 16 and 17. The PL at $3.7 \mathrm{eV}$ of $\mathrm{V}_{\mathrm{O}}^{+2}$ centers in $\mathrm{m}-\mathrm{HfO}_{2}$ is predicted to originate from radiative tunneling transition (RTT) between a $\mathrm{V}_{\mathrm{O}}^{+1}$ center and a self-trapped hole.

\section{METHODOLOGY}

\section{A. Computational details}

All simulations were carried out using periodic boundary conditions and the implementation of DFT in the CP2K software package. ${ }^{28}$ These calculations sample the Brillouin zone only at the $\Gamma$ point. To check the dependence of the results on the supercell size, the 216, 512 and 1000 atoms supercells were used for $\mathrm{MgO}$, and 96 and 324 atoms supercells for m- $\mathrm{HfO}_{2}$. Since we consider the 96 atom cell to be too small, we only analyze $\mathrm{HfO}_{2}$ defects and optical transitions for the 324 atom cell.

Charged defects in the periodic model are calculated using the neutralizing jellium background, as implemented in $\mathrm{CP} 2 \mathrm{~K}$. In this paper, we are mainly concerned with optical excitations of defects in particular charge states rather than their formation energies and transition levels, where charge corrections as well as potential alignment are important. TDDFT calculations include KS orbital energy differences, which are affected by the cell size as well as the extent of lattice distortion induced by charged defects. These effects are checked using periodic cells of increasing sizes.

The PBE0-TC-LRC exchange-correlation (XC) functional $^{29}$ was used with an exact exchange contribution of $32.5 \%$ for the $\mathrm{MgO}$ calculations and $25 \%$ for the $\mathrm{HfO}_{2}$ calculations. This functional is based on ordinary PBE0; ${ }^{30}$ however, HF exchange is only used for ranges up to a selected "truncation radius." Beyond the truncation radius, a long range correction (based on the spherically averaged PBE exchange hole $^{31}$ ) is applied. In this work, we use $6 \AA$ for the truncation radius in $\mathrm{MgO}$ and $4 \AA$ in $\mathrm{HfO}_{2}$.
The Goedecker-Tetter-Hutter (GTH) pseudopotentials and GTH MOLOPT basis sets were used for all atom species. ${ }^{32,33}$ In our setup, $\mathrm{Mg}$ has 2 valence electrons, whereas Hf and $\mathrm{O}$ both have 6 . All periodic cells had lattice parameters and optimized geometry such that forces were smaller than $0.023 \mathrm{eV} / \AA$. The effect of oxygen vacancies on the local structure was simulated by re-optimizing the cell geometry after the deletion of an oxygen atom. Lattice parameters were kept constant during optimization of the defective cells. Since $\mathrm{MgO}$ has an FCC structure, all oxygen atoms in the periodic cell are equivalent and it is not necessary to sample different sites. In $\mathrm{m}-\mathrm{HfO}_{2}, \mathrm{O}$ atoms can be either 3- or 4-coordinated by $\mathrm{Hf}$ ions. In this work, we focus only on $\mathrm{O}$ vacancies at $3 \mathrm{C}$ sites. Positive or negative oxygen vacancies were simulated by removal or addition of electrons from the defective cell and then re-optimizing the geometry.

To calculate optical transition energies and oscillator strengths, we use the well established linear response formulation of the TDDFT within the local adiabatic approximation. In this approximation, the XC-functional is simply one of the usual XC-functionals used for ground state DFT calculations. The detailed description of mathematical expressions implemented in CP2K is given in the Appendix.

The calculation of Hartree-Fock electron-repulsion integrals (ERIs) greatly increases the computational cost of hybrid functionals and renders the ground state DFT and TDDFT calculations infeasible for the system sizes considered here. For this reason, it is necessary to employ the auxiliary density matrix method (ADMM). ${ }^{34}$ This approximation utilizes a smaller and faster converging basis set, greatly speeding up the calculation of HF exact exchange. This allows us to use large supercells, which means we can more fully represent the structural relaxation induced by the presence of vacancies. Reducing the basis set quality can introduce errors in the HF exchange calculations. The key assumption of ADMM is outlined in Sec. II B.

\section{B. Auxiliary density matrix method}

In ground-state KS-DFT, the exact exchange energy, which is an essential component of hybrid XC-functionals, is expressed in terms of a density matrix $\mathrm{P}, 34$

$$
\mathrm{E}_{x}^{\mathrm{HF}}[\mathrm{P}]=\sum_{\mu \nu \lambda \sigma} \mathrm{P}_{\mu \lambda} \mathrm{P}_{\nu \sigma}(\mu \nu \mid \lambda \sigma)
$$

and Electron Repulsion Integrals (ERIs) over Gaussian basis functions $\{\chi\}$,

$$
(\mu \nu \mid \lambda \xi)=\int \chi_{\mu}^{*}(\mathbf{r}) \chi_{v}(\mathbf{r}) \frac{1}{\left|\mathbf{r}-\mathbf{r}^{\prime}\right|} \chi_{\lambda}^{*}\left(\mathbf{r}^{\prime}\right) \chi_{\xi}\left(\mathbf{r}^{\prime}\right) \mathrm{d} \mathbf{r} \mathrm{d} \mathbf{r}^{\prime} .
$$

The number of such integrals grows with the total number of atomic basis functions to the fourth power in a naive implementation that quickly becomes a bottleneck. However, the majority of these integrals are negligible. In particular, the inner product of two Gaussian functions centred on different atoms exponentially decays with the distance between these atoms. This effectively means that the number of nonnegligible ERIs scales quadratically with the system size for a given basis set. 
To take advantage of this fact, $\mathrm{CP} 2 \mathrm{~K}$ screens ERIs based on the Cauchy-Schwarz inequality,

$$
(\mu \nu \mid \lambda \sigma) \leq \sqrt{(\mu \nu \mid \mu \nu)} \sqrt{(\lambda \xi \mid \lambda \xi)}
$$

and ignores the integrals which are less than the given threshold. Screening based additionally on the size of the density matrix element of the pair of orbitals in the ground state wave function can further reduce this to linear scaling. However, even in this case, evaluation of ERIs still remains a challenging task due to a large prefactor. This prefactor becomes much larger when the basis set is augmented with diffuse functions because these functions have a slower long-range decay. This problem also affects the MOLOPT basis sets commonly used in $\mathrm{CP} 2 \mathrm{~K},{ }^{33}$ as they contain significant numbers of (contracted) diffuse basis functions.

The Auxiliary Density Matrix Method (ADMM) addresses this problem by computing ERIs using a small auxiliary basis set with rapidly decaying basis functions $\{\tilde{\chi}\}$. An approximate auxiliary density matrix $\tilde{P}$ is then constructed by fitting the density matrix in the primary basis set $(P)$,

$$
\tilde{\mathrm{P}}=\mathrm{OPO}^{\mathrm{T}} \text {, }
$$

using a projector from the primary basis set onto the auxiliary basis set,

$$
\begin{gathered}
\mathrm{O}=\tilde{\mathrm{S}}^{-1} \mathrm{U}, \\
\tilde{\mathrm{S}}_{\mu \nu}=\left\langle\tilde{\chi}_{\mu} \mid \tilde{\chi}_{\nu}\right\rangle, \quad \mathrm{U}_{\mu v}=\left\langle\tilde{\chi}_{\mu} \mid \chi_{v}\right\rangle .
\end{gathered}
$$

Optionally, the ground-state auxiliary density matrix can be purified using a number of techniques ${ }^{34}$ to ensure that all properties of a true density matrix are met. We have not implemented purification for use with TDDFT methods, as a response density matrix $P^{(1)}$-which appears in Eq. (1) instead of the ground-state density matrix-does not satisfy all conditions for a pure density matrix (see the Appendix for further details).

ADMM also assumes that the difference between exactexchange energies computed using primary and auxiliary basis sets has (semi-)local nature and thus can be well described using some reference LDA or generalised gradient approximation (GGA) exchange functional $\left(E_{\mathrm{x}}^{\mathrm{DFT}}\right)$. It naturally leads to the trivial expression for the approximate exact-exchange energy in the primary basis set,

$$
\mathrm{E}_{\mathrm{x}}^{\mathrm{HF}}[\mathrm{P}] \approx \mathrm{E}_{\mathrm{x}}^{\mathrm{HF}}[\tilde{\mathrm{P}}]+\left(\mathrm{E}_{\mathrm{x}}^{\mathrm{DFT}}[\mathrm{P}]-\mathrm{E}_{\mathrm{x}}^{\mathrm{DFT}}[\tilde{\mathrm{P}}]\right)
$$

Differentiation of the above expression with respect to the density matrix gives the following contribution to the KohnSham matrix: 35

$$
\mathrm{K}_{\mathrm{x}}^{\mathrm{HF}} \approx \mathrm{F}_{\mathrm{x}}^{\mathrm{DFT}}+\mathrm{O}^{\mathrm{T}}\left(\tilde{\mathrm{K}}_{\mathrm{x}}^{\mathrm{HF}}-\tilde{\mathrm{F}}_{\mathrm{x}}^{\mathrm{DFT}}\right) \mathrm{O},
$$

where

$$
\begin{aligned}
\left(\mathrm{F}_{\mathrm{x}}^{\mathrm{DFT}}\right)_{\alpha \beta} & =\int \chi_{\alpha}^{*}(\mathbf{r}) v_{x}[\mathrm{P}](\mathbf{r}) \chi_{\beta}(\mathbf{r}) \mathrm{d} \mathbf{r}, \\
\left(\tilde{F}_{\mathrm{x}}^{\mathrm{DFT}}\right)_{\mu \lambda} & =\int \tilde{\chi}_{\mu}^{*}(\mathbf{r}) v_{x}[\tilde{\mathrm{P}}](\mathbf{r}) \tilde{\chi}_{\lambda}(\mathbf{r}) \mathrm{d} \mathbf{r} \\
\left(\tilde{\mathrm{K}}_{\mathrm{x}}^{\mathrm{HF}}\right)_{\mu \lambda} & =\sum_{\nu \sigma} \tilde{P}_{\nu \sigma}(\tilde{\mu} \tilde{\nu} \mid \tilde{\lambda} \tilde{\sigma})
\end{aligned}
$$

and $v_{\mathrm{x}}$ is the (semi-)local reference exchange potential as a functional of the electron density.

\section{RESULTS AND DISCUSSION}

\section{A. Properties of perfect crystals}

We start from considering the calculated bulk properties for different cell sizes of $\mathrm{MgO}$ and $\mathrm{m}-\mathrm{HfO}_{2}$. All calculations are performed sampling the Brillouin zone at the $\Gamma$ point. The bandgap energies are calculated as the difference of KohnSham (KS) energies and using TDDFT. The results shown in Tables I and II for $\mathrm{MgO}$ and $\mathrm{m}-\mathrm{HfO}_{2}$, respectively, demonstrate good agreement of lattice parameters with experimental reports. However the band gap of $\mathrm{HfO}_{2}$ is slightly overestimated. We note that in both systems, the TDDFT calculated optical bandgap is lower than the KS bandgap. This is because, unlike in GGA-based TDDFT, in hybrid functional based TDDFT, there is electron-hole interaction. ${ }^{36}$ Excitation between delocalized band states creates an electron-hole pair which is confined within the simulation cell and thus has an artificially high electron-hole interaction energy. This explains why the difference between the KS and TDDFT bandgap is greatest for small simulation cells where the electron-hole pair is more confined and thus the electron-hole interaction is greater.

\section{B. Optical transitions of $O$ vacancies}

The removal of a neutral oxygen atom in the $\mathrm{MgO}$ or $\mathrm{m}-\mathrm{HfO}_{2}$ periodic cell leaves behind a neutral oxygen vacancy, $\mathrm{V}_{\mathrm{O}}^{0}$. Two electrons localize on the vacancy (see Fig. 2). These localized electrons occupy two degenerate mid-gap levels (one state for each spin) which are located $4.45 \mathrm{eV}$ below the conduction band minimum (CBM) in $\mathrm{MgO}$ and $3.3 \mathrm{eV}$ below the $\mathrm{CBM}$ in $\mathrm{HfO}_{2}$. In general, the vacancy perturbs the electronic

TABLE I. The lattice parameters $(\AA)$ and bandgap values (eV) for bulk MgO. The optical bandgap (BG) is calculated using TDDFT. Cell sizes are given in number of atoms.

\begin{tabular}{lllcc}
\hline \hline & \multicolumn{4}{c}{ Cell size } \\
\cline { 2 - 5 } & 216 & 512 & 1000 & Exp. \\
\hline |a| & 4.21 & 4.21 & 4.21 & $4.211^{37}$ \\
KS BG & 7.7 & 7.7 & 7.8 & $\ldots$ \\
Optical BG & 7.1 & 7.4 & 7.5 & $7.78^{6}$ \\
\hline \hline
\end{tabular}

TABLE II. The lattice parameters $(\AA)$ and bandgap energies (eV) calculated for bulk $\mathrm{m}-\mathrm{HfO}_{2}$. The optical bandgap (BG) is calculated using TDDFT.

\begin{tabular}{lccc}
\hline \hline & \multicolumn{3}{c}{ Cell size } \\
\cline { 2 - 4 } & 96 & 324 & Exp. \\
\hline$|\mathbf{a}|$ & 5.08 & 5.07 & $5.117^{38}$ \\
$|\mathbf{b}|$ & 5.13 & 5.13 & $5.18^{38}$ \\
$|\mathbf{c}|$ & 5.25 & 5.24 & $5.29^{38}$ \\
$\beta$ (degrees) & 99.12 & 99.11 & $99.22^{38}$ \\
KS BG & 6.51 & 6.66 & $\ldots$ \\
Optical BG & 6.0 & 6.1 & $5.68^{39}$ \\
\hline \hline
\end{tabular}


(A)

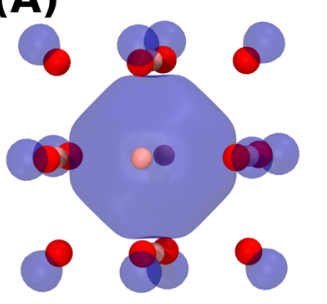

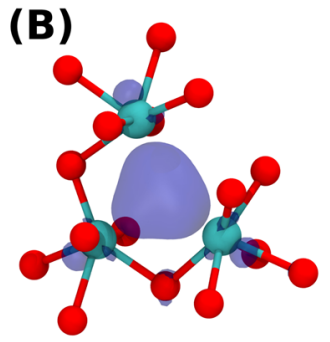

FIG. 2. The nearest-neighbor environment of neutral O-vacancy in $\mathrm{MgO}$ (a) and $\mathrm{HfO}_{2}$ (b). An isosurface of the wavefunction of the doubly occupied defect state is shown in blue. $\mathrm{Mg}$ ions are colored in peach, $\mathrm{Hf}$ in cyan, and $\mathrm{O}$ in red.

structure, causing quasi-local states to appear in $\mathrm{VB}$ and $\mathrm{CB}$, as shown in Fig. 1.

Structural relaxation around the neutral vacancy is small since the Coulomb interaction between the vacancy and nearby ions is similar to the Coulomb interaction between an oxygen ion and its neighbors in the bulk. Charging the vacancy causes greater structural reorganization. For example, charging the $\mathrm{V}_{\mathrm{O}}^{0}$ defect to the +1 state in $\mathrm{MgO}$ results in the nearestneighbor Mg ions moving outward by $0.11 \AA$ (see Table III). The calculated displacements are in good agreement with those derived from the analysis of EPR spectra of the $\mathrm{V}_{\mathrm{O}}^{+1}$ center in Ref. 40.

In this study, we look at the $0,+1$, and +2 charge states of $\mathrm{O}$ vacancies in $\mathrm{MgO}$ and $\mathrm{HfO}_{2}$. In the +1 charge state, the vacancy is paramagnetic. Removing an electron from $\mathrm{V}_{\mathrm{O}}^{0}$ (and then relaxing) to create a $\mathrm{V}_{\mathrm{O}}^{+1}$ defect splits the doubly occupied state in the bandgap into two states which are energetically separated. In $\mathrm{MgO}$, for example, the occupied $\alpha$ spin state sits $5.2 \mathrm{eV}$ below the CBM, whereas the $\beta$ spin state is unoccupied and is higher in the bandgap (see Fig. 1). The nature of the transition in the +1 state therefore depends on the spin channel: alpha spin excitations will involve a transition from a mid gap state into CB states, whereas a beta spin transition involves a VB electron being promoted into a gap state.

TABLE III. The displacements (in $\AA$ ) of the ions surrounding an oxygen vacancy in $\mathrm{MgO}$ and $\mathrm{HfO}_{2} . \Delta_{\mathrm{Mg}}, \Delta_{\mathrm{Hf}}$, and $\Delta_{\mathrm{O}}$. In MgO, high symmetry means that all $\mathrm{NN} \mathrm{Mg}$ ions are displaced in equal amounts, as are all NNN oxygen ions. In $\mathrm{HfO}_{2}$, however, the surrounding $\mathrm{Hf}$ and $\mathrm{O}$ ions are not displaced equally. For $\mathrm{HfO}_{2}$, the range of displacements is shown.

\begin{tabular}{lcc}
\hline \hline & Ionic displacements & \\
\hline $\mathrm{MgO}$ & $\Delta_{\mathrm{Mg}}$ & $\Delta_{\mathrm{O}}$ \\
\hline $\mathrm{V}_{\mathrm{O}}^{0}$ & 0.02 & 0.01 \\
$\mathrm{~V}_{\mathrm{O}}^{+1}$ & 0.11 & 0.03 \\
$\mathrm{~V}_{\mathrm{O}}^{+2}$ & 0.20 & 0.08 \\
\hline $\mathrm{HfO}_{2}$ & $\Delta_{\mathrm{Hf}}$ & $\Delta_{\mathrm{O}}$ \\
\hline $\mathrm{V}_{\mathrm{O}}^{0}$ & $0.01-0.08$ & $0.02-0.09$ \\
$\mathrm{~V}_{\mathrm{O}}^{+1}$ & $0.09-0.11$ & $0.04-0.18$ \\
$\mathrm{~V}_{\mathrm{O}}^{+2}$ & $0.19-0.24$ & $0.07-0.39$ \\
\hline \hline
\end{tabular}

The wavefunctions of excited states calculated using TDDFT are linear combinations of determinants where $\Psi_{i}^{a}$ is a singly excited wave-function, whose $i$ th occupied Molecular Orbital (MO) is replaced by the ath virtual MO (see the Appendix). Often one excitation $\Psi_{i}^{a}$ dominates and will be used to qualitatively represent the excited state in further discussion.

The lowest energy transition of the $\mathrm{MgO} \mathrm{V}_{\mathrm{O}}^{0}$ defect has zero oscillator strength because the singly occupied defect state and the LUMO state dominating this transition have similar cubic symmetry (see Fig. 2) analogous to hydrogenic 1s and 2 s orbitals. The next three higher energy transitions have equal excitation energies and are responsible for the main excitation peak seen in $\mathrm{V}_{\mathrm{O}}^{0}$ defects in $\mathrm{MgO}$ [Fig. 3(a)]. They correspond to the excitation into a degenerate p-like state.

$\mathrm{V}_{\mathrm{O}}^{0}$ center in $\mathrm{m}-\mathrm{HfO}_{2}$ has lower symmetry and the TDDFT calculations predict a broader absorption spectrum with an onset at $2.5 \mathrm{eV}$ and then with higher energy peaks at $3.0 \mathrm{eV}$ and $3.5 \mathrm{eV}$. Also, unlike in $\mathrm{MgO}$, the $\mathrm{V}_{\mathrm{O}}^{0}$ excitation in $\mathrm{HfO}_{2}$ is from the vacancy state into the states delocalized at the bottom of the conduction band, hence much lower oscillator strengths of these transitions. The calculated spectra of the $\mathrm{V}_{\mathrm{O}}^{0}$ in $\mathrm{HfO}_{2}$ are plotted in Fig. 3d. We note that the predicted transition energies are in good agreement with the results of embedded cluster calculations in Ref. 26.

In the +1 charge state, transitions in the $\alpha$ and $\beta$ spin channels are no longer equivalent. In $\mathrm{MgO}$, the absorption energies in both spin channels have been predicted to be close to one another, ${ }^{13}$ making the interpretation of optical absorption spectra difficult. Here, however, we predict that the $\beta$ spin excitation has higher energy [Fig. 3(b)]. This should lead to asymmetry in high energy part of the optical absorption spectrum of the $\mathrm{V}_{\mathrm{O}}^{+1}$ center and can explain the asymmetric line shape observed in Ref. 7 . There will also be qualitative differences between the two types of excitation. Excitations in the $\alpha$ channel will excite an electron from the gap state into $\mathrm{CB}$ states, similar to the $\mathrm{V}_{\mathrm{O}}^{0}$ defect. Excitations in the $\beta$ channel, however, will release holes into the valence band. It is then possible for these holes to be trapped onto V-centers. ${ }^{13}$

We note that our results do not support the prediction ${ }^{15}$ that the $\beta$ absorption energy should be much lower than the $\alpha$ spin absorption energy $(\approx 3.6 \mathrm{eV})$. It is possible that the $3.6 \mathrm{eV}$ absorption band emerges due to constraint on the $\mathrm{V}_{\mathrm{O}}^{+1}$ center relaxation imposed by small periodic cells used in Ref. 15. Indeed, charging the $\mathrm{V}_{\mathrm{O}}^{0}$ center to the +1 state but not allowing the structure to relax brings the main $\beta$-spin absorption energy down to $3.5 \mathrm{eV}$. This is not surprising as the defectinduced lattice distortion strongly affects the positions of quasi-local states in the valence band responsible for $\beta$ transitions. We therefore find that failure to allow the charged defect to fully relax can change absorption energies by as much as $2 \mathrm{eV}$.

In $\mathrm{HfO}_{2}$, optical excitations in the $\alpha$ and $\beta$ spin channels have different energies. To illustrate why, we can look at the electronic structure of the $\mathrm{V}_{\mathrm{O}}^{+1}$ defect in $\mathrm{HfO}_{2}$. The alpha HOMO-LUMO separation is calculated to be $3.6 \mathrm{eV}$, whereas the beta HOMO-LUMO separation is 
(A)

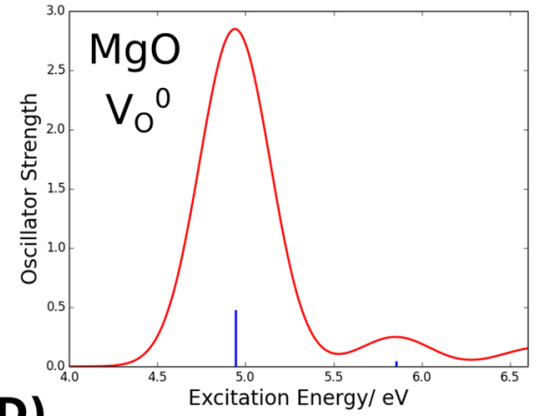

(D)

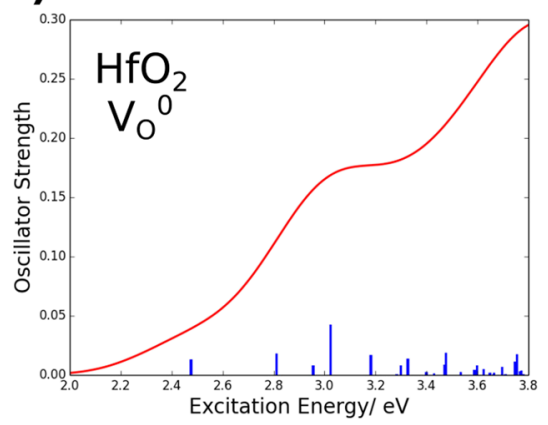

(B)

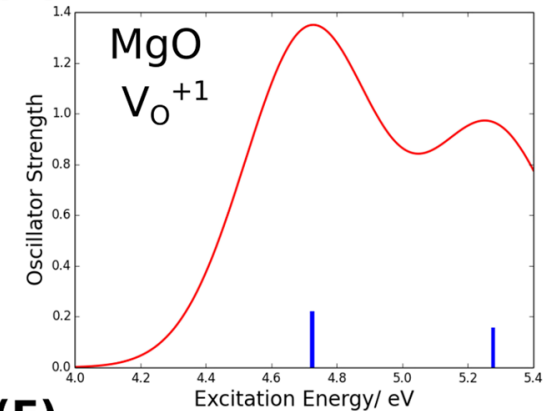

(E)

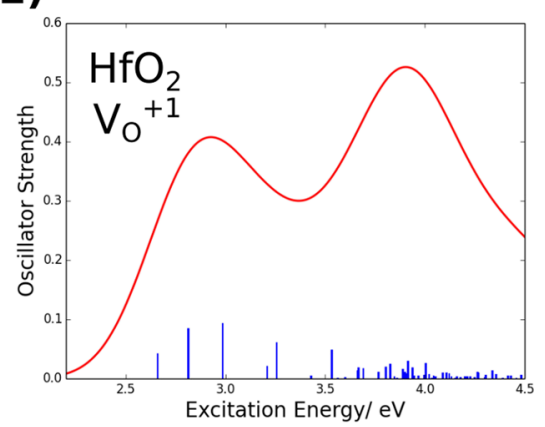

(C)

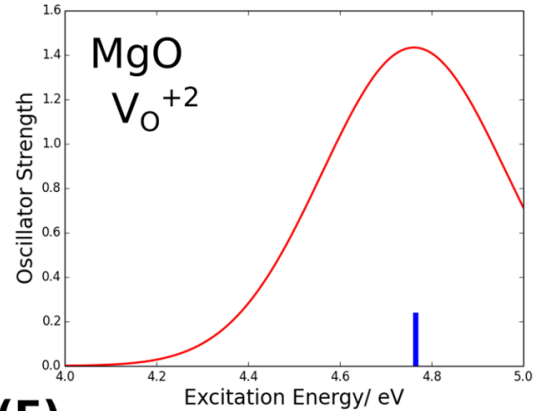

(F)

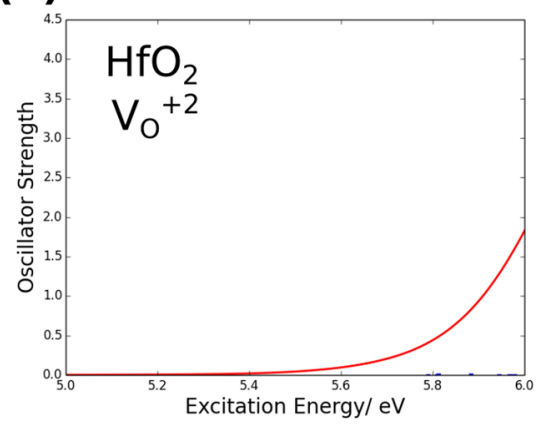

FIG. 3. Optical absorption spectra of oxygen vacancies in $\mathrm{MgO}$ and $\mathrm{m}-\mathrm{HfO}_{2}$. (a) is the $\mathrm{V}_{\mathrm{O}}^{0}$ defect in $\mathrm{MgO}$. There is strong absorption peaking at $4.85 \mathrm{eV}$. This corresponds to a transition between an s-like and a p-like states. (b) shows transitions in the $\mathrm{V}_{\mathrm{O}}^{+1}$ center in $\mathrm{MgO}$. The lower energy absorption peaks at 4.70 and comes from excitation of an $\alpha$ spin electron in a gap-state into CB states. The higher energy absorption peaks at $5.26 \mathrm{eV}$ and comes from excitation of electrons in VB states into the unoccupied state in the gap. (c) is the $\mathrm{V}_{\mathrm{O}}^{+2}$ defect in $\mathrm{MgO}$. (d), (e), and (f) correspond to the $\mathrm{V}_{\mathrm{O}}^{0}, \mathrm{~V}_{\mathrm{O}}^{+1}$ and $\mathrm{V}_{\mathrm{O}}^{+2}$ centers in $\mathrm{m}-\mathrm{HfO}_{2}$, respectively. All the transitions represented by (e) are from excitations in the alpha channel. Individual transitions (without smearing) are shown by delta functions, and in (f), these transitions are too small to be seen. The smeared spectra is plotted using Gaussian smearing of $\sigma=0.2 \mathrm{eV}$.

calculated to be $5.6 \mathrm{eV}$ (very close to the optical bandgap value). Thus, when irradiating $\mathrm{HfO}_{2}$ with photons of subbandgap energy, we expect the $\mathrm{V}_{\mathrm{O}}^{+1}$ spectrum to be dominated by $\alpha$ spin transitions (transitions from the vacancy state into the CB states, i.e., type IV in Fig. 1). Indeed we predict that the first optical absorption peak occurs at $2.9 \mathrm{eV}$ and corresponds to the transition of the alpha-spin electron from the gap-state into a quasi-local state in the $\mathrm{CB}$. All the higher energy peaks up to bandgap energy also correspond to excitations of the $\alpha$-spin electron out of the gap-state and into higher energy $\mathrm{CB}$ states. Excitations in the $\beta$ spin channel are only attainable once we arrive at excitation energies comparable with the bandgap.

In both materials, the $\mathrm{V}_{\mathrm{O}}^{+2}$ defect produces an unoccupied state high in the bandgap as well as resonant states in the $\mathrm{CB}$ (Fig. 1). In $\mathrm{MgO}$, the optical absorption of the $\mathrm{V}_{\mathrm{O}}^{+2}$ center has been predicted ${ }^{26}$ to be close to the $\mathrm{V}_{\mathrm{O}}^{0}$ and $\mathrm{V}_{\mathrm{O}}^{+1}$ defects. It is dominated by transitions from the defect-induced states in the valence band into the LUMO defect state located close to the bottom of the CB (type III in Fig. 1). Therefore, all of the $\mathrm{V}_{\mathrm{O}}$ defects in $\mathrm{MgO}$ are predicted to have optical absorption peaks near $5 \mathrm{eV}$. This explains why it is so difficult to distinguish between different charge states of the $\mathrm{V}_{\mathrm{O}}$ defect in $\mathrm{MgO}$ using optical absorption experiments alone.
In $\mathrm{HfO}_{2}$, the optical transition energies from the valence band into the $\mathrm{V}_{\mathrm{O}}^{+2}$ defect are comparable to the bandgap energy and are predominantly of type II in Fig. 1. Optical experiments conducted on $\mathrm{HfO}_{2}{ }^{24}$ have detected a $5.2 \mathrm{eV}$ absorption peak which has been attributed to the presence of oxygen vacancies. We predict that the doubly positively charged oxygen vacancy $\left(\mathrm{V}_{\mathrm{O}}^{+2}\right)$ has an optical absorption peak at $5.3 \mathrm{eV}$, which is in good agreement with this detected absorption band. We note that the position of the peak predicted in this work is at higher photon energy than $4.94 \mathrm{eV}$ calculated in Ref. 26 using TDDFT in a relatively small embedded cluster. This is consistent with our observation that full account of defect-induced lattice distortion for charged defects is important for predicting correct optical absorption energies.

\section{Photoluminescence energies}

As was mentioned above, MgO has a well-established PL band at $2.3 \mathrm{eV}$ attributed to the $\mathrm{V}_{\mathrm{O}}^{0}$ center. $^{8-10,16}$ The lifetime of this luminescence is much longer than that for the $\mathrm{V}_{\mathrm{O}}^{+}$center, which is caused by a single-electron transition in the doublet state. Therefore the $2.3 \mathrm{eV}$ luminescence of the $\mathrm{V}_{\mathrm{O}}^{0}$ center could be due to a partially allowed $2 \mathrm{~s} \rightarrow 1 \mathrm{~s}$ type 
and/or triplet-to-singlet transition. The kinetics of this PL is, however, much more complicated than that of the $\mathrm{V}_{\mathrm{O}}^{0}$ center in $\mathrm{CaO}$, where the lowest relaxed excited state has been shown to have a tetragonal (100) triplet state with fast spin-lattice relaxation (see, for example, Ref. 41). The PL kinetics in $\mathrm{MgO}$ is strongly affected by the presence of $\mathrm{H}$ and other impurities and depends on sample preparation. ${ }^{8,16}$ Semi-empirical ${ }^{16}$ calculations suggest that tetragonal singlet and triplet states are both located very close to the bottom of the $\mathrm{CB}$ and that there are two minima in the relaxed excited state corresponding to ${ }^{3} \mathrm{~T}_{1 u}$ and ${ }^{3} \mathrm{~A}_{1 g}$ configurations, respectively. The predicted PL energies corresponding to transitions from these two minima are equal to $2.9 \mathrm{eV}$ and $2.2 \mathrm{eV}$, respectively. The PL peaking at $2.9 \mathrm{eV}$ has been observed experimentally ${ }^{42}$ but has an excitation energy at about $7 \mathrm{eV}$. It has been concluded that the PL peaks at $2.3 \mathrm{eV}$ and $2.9 \mathrm{eV}$ may involve both transitions intrinsic to the $V_{O}^{0}$ center and those caused by electron transfer from impurities. ${ }^{8,16}$ We note that the dynamics of similar PL of $\mathrm{F}$ centers in alkali halides has been studied in detail. ${ }^{43}$ In that case, the transition is allowed due to mixing of $2 \mathrm{~s}$ and $2 \mathrm{p}$ terms, with $2 \mathrm{~s}$ having the lower energy in the relaxed excited state. 43

To shed more light on the nature of excited states, we have calculated the triplet excited states of the $\mathrm{V}_{\mathrm{O}}^{0}$ center in $\mathrm{MgO}$ using the so-called $\triangle \mathrm{SCF}$ method. This computational procedure employs a non-Aufbau occupation of the triplet state in a dielectric to converge the KS equations to an excited state. ${ }^{44,59}$ The total energy and geometry of the triplet excited state of a defect are calculated self-consistently. This approach allows us to predict defect geometries in the electronically excited state (the feature still unavailable in TDDFT in CP2K). The lowest energy triplet state has ${ }^{3} \mathrm{~A}_{1 g}$ symmetry, and the calculated luminescence energy from this state is $2.0 \mathrm{eV}$. A higher energy triplet state has a geometry formed from a combination of $\mathrm{A}_{1 g}$ and $\mathrm{T}_{2 g}$ displacement modes. The calculated PL energy from this state is $2.9 \mathrm{eV}$. Thus our calculations support the PL model suggested in earlier studies 8,16 with surprising agreement of calculated PL energies.

We used the same approach to investigate the luminescence of the neutral oxygen vacancy in $\mathrm{m}-\mathrm{HfO}_{2}$. In the case of optical excitation of the $\mathrm{V}_{\mathrm{O}}^{0}$ defect, the electron-hole pair remains bound to the vacancy. The predicted triplet-singlet PL energy is $0.8 \mathrm{eV}$.

The situation is, however, more intriguing in the case of the $\mathrm{V}_{\mathrm{O}}^{+2}$ center. It produces an unoccupied state inside the gap close to the bottom of the $\mathrm{CB}$ as well as quasi-local states in the $\mathrm{VB}$ and $\mathrm{CB}$ in both $\mathrm{MgO}$ and $\mathrm{m}-\mathrm{HfO}_{2}$. In alkali halides, a singly positively charged anion vacancy (also called an $\alpha$-center) has a qualitatively similar electronic structure and a characteristic luminescence, which is close in energy to the exciton luminescence (the so-called $\alpha$-luminescence) and is attributed to exciton perturbed by the vacancy. ${ }^{45}$ The relaxed excited state of this center can be also viewed as an electron transferred into the vacancy and a hole trapped next to the vacancy. ${ }^{46}$ Such luminescence has not been observed for the $\mathrm{V}_{\mathrm{O}}^{+2}$ center in $\mathrm{MgO}$ because free excitons in $\mathrm{MgO}$ are very mobile and get trapped by impurities. Since excitons and holes have been suggested to self-trap in $\mathrm{m}-\mathrm{HfO}_{2},{ }^{47-49}$ one could expect creation of $\alpha$-type luminescence in this material. Recent experiments ${ }^{27}$ suggest that $3.6 \mathrm{eV}$ luminescence excited by $5.3 \mathrm{eV}$ photons could be due to charged vacancies in $\mathrm{m}-\mathrm{HfO}_{2}$.

Using the $\triangle \mathrm{SCF}$ method, we investigated the triplet excited state of the $\mathrm{V}_{\mathrm{O}}^{+2}$ defect in $\mathrm{HfO}_{2}$. It is found that the relaxed triplet configuration of the $\mathrm{V}_{\mathrm{O}}^{+2}$ center has an electron localized into the vacancy (producing a $\mathrm{V}_{\mathrm{O}}^{+1}$ center) and a hole localized in a polaron state elsewhere in the supercell. Holes have been predicted to self-trap in $\mathrm{m}-\mathrm{HfO}_{2}$ at low temperatures. ${ }^{48,50}$ Due to repulsion with the positively charged vacancy, the hole cannot sit close to the vacancythe closest stable separation we find between the hole polaron and the vacancy is approximately $5 \AA$ (see Fig. 4). Therefore the localized hole and vacancy can be viewed as two separated defects. In such a system, luminescence due to electron-hole recombination can occur when the electron in the $\mathrm{V}^{+1}$ defect tunnels into the hole polaron via a radiative tunneling transition (RTT). The RTT recombination luminescence of spatially well-separated electron and hole defects has been studied in semiconductors ${ }^{51}$ and ionic crystals. ${ }^{52}$ It usually occurs between ground electronic states of donor and acceptor.

We predict the photon energy of this emission to be $3.7 \mathrm{eV}$. Unlike in semiconductors, ${ }^{51}$ the emission energy does not depend on the defect separation in the range of up to $11 \AA$ (this upper limit originates in the finite size of the supercell) due to dielectric screening in the high- $k$ material. In Ref. 53, a range of luminescence peaks between 2.0 and $3.7 \mathrm{eV}$, attributed to oxygen vacancies, have been detected at approximately $7 \mathrm{~K}$ using cathodoluminescence and optical luminescence. In another optical experiment, ${ }^{47}$ conducted at $10 \mathrm{~K}$, weak emission in the range of 2.2 to $3.6 \mathrm{eV}$ has been detected. A recent study ${ }^{27}$ also found luminescence peaking near $3.6 \mathrm{eV}$, again linked to oxygen vacancies. These results are consistent with the optical absorption of the $\mathrm{V}_{\mathrm{O}}^{+2}$ defect and the

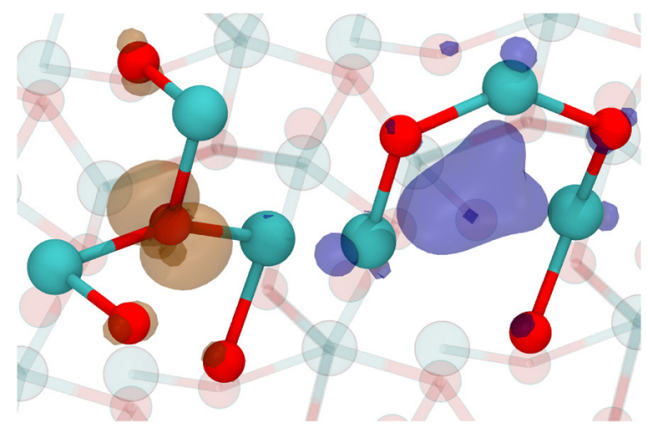

FIG. 4. The relaxed triplet configuration of the $\mathrm{V}_{\mathrm{O}}^{+2}$ defect. An electron localises around the vacancy (to create $\mathrm{a} \mathrm{V}_{\mathrm{O}}^{+1}$ defect) and a hole self-traps elsewhere in the lattice. A range of separations between hole and vacancy are possible. Here, the closest separation configuration is shown. Transparent surfaces show the $|\psi|^{2}$ of the hole (orange) and electron (blue). The iso-surface value is 0.007 . The nearest neighbor atoms of each defect are highlighted. 
predicted RTT luminescence energy. However, further studies are required to elucidate the nature of experimentally observed peaks.

\section{CONCLUSIONS}

To conclude, we used a newly implemented TDDFT algorithm in $\mathrm{CP} 2 \mathrm{~K}$ to conduct optical absorption calculations on oxygen vacancies in $\mathrm{MgO}$ and $\mathrm{HfO}_{2}$. Using a range of periodic cells, we demonstrate that failure to properly describe the geometric structure of a defect significantly affects the predicted absorption energies, potentially by several eV. We confirm the existing models of the nature of optical absorption and photoluminescence of $\mathrm{O}$ vacancies in $\mathrm{MgO}$ and $\mathrm{HfO}_{2}$ and predict that the PL at $3.7 \mathrm{eV}$ in $\mathrm{HfO}_{2}$ excited at $5.2 \mathrm{eV}$ could originate from radiative tunneling transitions between a $\mathrm{V}^{+1}$ center and hole polaron created by this excitation. The results of our calculations are in good agreement with the available experimental data and shed light on the nature of optical absorption and luminescence peaks in these materials. Therefore the use of TDDFT with a hybrid functional, which strikes a good balance between accuracy and computational efficiency, is appropriate for the prediction of the optical properties of defects in other solid state systems.

\section{ACKNOWLEDGMENTS}

We acknowledge funding provided by the UK Engineering and Physical Sciences Research Council (EPSRC) under Grant Nos. EP/K01739X/1 and EP/P013503/1 and by the Leverhulme Trust RPG-2016-135. J.S. is funded by EPSRC Grant No. EP/G036675/1 to the Center for Doctoral Training. Computer facilities on the ARCHER UK National Supercomputing Service have been provided via the UKs HPC Materials Chemistry Consortium (EPSRC Grant No. EP/L000202). Part of this work was funded under the embedded CSE programme of the ARCHER UK National Supercomputing Service (http://www.archer.ac.uk).

\section{APPENDIX: LINEAR RESPONSE TIME-DEPENDENT DENSITY FUNCTIONAL THEORY}

In the linear response (LR) formulation, TDDFT equations can be cast in the form of a non-Hermitian eigenproblem, ${ }^{54}$

$$
\left(\begin{array}{cc}
A & B \\
B^{*} & A^{*}
\end{array}\right)\left(\begin{array}{l}
X_{p} \\
Y_{p}
\end{array}\right)=\omega_{p}\left(\begin{array}{cc}
1 & 0 \\
0 & -1
\end{array}\right)\left(\begin{array}{l}
X_{p} \\
Y_{p}
\end{array}\right),
$$

where $\left(\mathrm{X}_{p}, \mathrm{Y}_{p}\right)$ is an eigenvector and $\omega_{p}$ is a corresponding transition energy. In terms of Kohn-Sham orbitals $\{\phi\}$, the elements of the matrices $A$ and $B$ can be written as ${ }^{55}$

$$
\begin{aligned}
\mathrm{A}_{i a \sigma, j b \tau}= & \mathrm{A}_{i a \sigma, j b \tau}^{\mathrm{E}}+\mathrm{A}_{i a \sigma, j b \tau}^{\mathrm{J}}+\mathrm{c}_{\mathrm{HFX}} \mathrm{A}_{i a \sigma, j b \tau}^{\mathrm{HFX}}+\mathrm{A}_{i a \sigma, j b \tau}^{\mathrm{XC}} \\
= & \delta_{i j} \delta_{a b} \delta_{\sigma \tau}\left(\epsilon_{a \sigma}-\epsilon_{i \sigma}\right)+\left(i_{\sigma} a_{\sigma} \mid j_{\tau} b_{\tau}\right) \\
& -c_{\mathrm{HFX}} \delta_{\sigma \tau}\left(i_{\sigma} j_{\sigma} \mid a_{\tau} b_{\tau}\right)+\left(i_{\sigma} a_{\sigma}\left|f_{\mathrm{xc} ; \sigma \tau}\right| j_{\tau} b_{\tau}\right), \\
\mathrm{B}_{i a \sigma, j b \tau}= & \left(i_{\sigma} a_{\sigma} \mid b_{\tau} j_{\tau}\right)-c_{\mathrm{HFX}} \delta_{\sigma \tau}\left(i_{\sigma} b_{\sigma} \mid a_{\tau} j_{\tau}\right) \\
& +\left(i_{\sigma} a_{\sigma}\left|f_{\mathrm{xc} ; \sigma \tau}\right| b_{\tau} j_{\tau}\right) .
\end{aligned}
$$

Here $A^{E}, A^{J}, A^{H F X}$, and $A^{\mathrm{XC}}$ denote orbital energy difference, electron-hole Coulomb, exact-exchange, and exchangecorrelation (XC) terms, respectively, while notations $\left(i_{\sigma} a_{\sigma} \mid j_{\tau} b_{\tau}\right)$ and $\left(i_{\sigma} a_{\sigma}\left|f_{\mathrm{xc} ; \sigma \tau}\right| j_{\tau} b_{\tau}\right)$ stand for four-centre electron repulsion integrals (ERIs) and XC-integrals,

$$
\begin{gathered}
\left(i_{\sigma} a_{\sigma} \mid j_{\tau} b_{\tau}\right)=\int \phi_{i \sigma}^{*}(\mathbf{r}) \phi_{a \sigma}(\mathbf{r}) \frac{1}{\left|\mathbf{r}-\mathbf{r}^{\prime}\right|} \phi_{j \tau}^{*}\left(\mathbf{r}^{\prime}\right) \phi_{b \tau}\left(\mathbf{r}^{\prime}\right) \mathrm{d} \mathbf{r} \mathrm{d} \mathbf{r}^{\prime}, \quad(\mathrm{A} 4) \\
\left(i_{\sigma} a_{\sigma}\left|f_{\mathrm{xc} ; \sigma \tau}\right| j_{\tau} b_{\tau}\right)=\int \phi_{i \sigma}^{*}(\mathbf{r}) \phi_{a \sigma}(\mathbf{r}) f_{\mathrm{xc} ; \sigma \tau}\left(\mathbf{r}, \mathbf{r}^{\prime}\right) \phi_{j \tau}^{*}\left(\mathbf{r}^{\prime}\right) \phi_{b \tau}\left(\mathbf{r}^{\prime}\right) \mathrm{d} \mathbf{r} \mathrm{d} \mathbf{r}^{\prime} .
\end{gathered}
$$

We use the following index convention: $i$ and $j$ label occupied orbitals, $a$ and $b$ stand for virtual orbitals, and $\sigma$ and $\tau$ refer to spin components. Besides, the quantity $\epsilon_{i \sigma}$ stands for the $i$ th Kohn-Sham orbital energy, and $f_{\mathrm{xc} ; \sigma \tau}\left(\mathbf{r}, \mathbf{r}^{\prime}\right)$ is a response $\mathrm{XC}$-kernel. The explicit expression for the $\mathrm{A}^{\mathrm{XC}}$ term in Eq. (A2) is given in the adiabatic approximation which postulates independence of the XC-functional on time. As such, XC-kernel becomes the second functional derivative of the $\mathrm{XC}$-functional $\left(E_{\mathrm{xc}}\right)$ over the ground-state electron density $\left(\rho^{(0)}\right),{ }^{56}$

$$
f_{\mathrm{xc} ; \sigma \tau}\left(\mathbf{r}, \mathbf{r}^{\prime}\right)=\left.\frac{\delta^{2} E_{\mathrm{xc}}[\rho](\mathbf{r})}{\delta \rho_{\sigma}\left(\mathbf{r}^{\prime}\right) \delta \rho_{\tau}\left(\mathbf{r}^{\prime}\right)}\right|_{\rho=\rho^{(0)}} .
$$

It is important to note that in case of a hybrid exchange functional, the exact-exchange term is excluded from the XCfunctional prior taking its functional derivative. For example, in case of the ordinary PBE0 XC-functional ${ }^{30}$ which contains $100 \%$ PBE correlation, 75\% PBE exchange, and 25\% exactexchange energies, only the first two terms contribute toward the XC-kernel.

As is customary, we also use the Tamm-Dancoff approximation (TDA) ${ }^{57}$ which amounts to setting all the elements of the matrix B in Eq. (A1) to zero. This simplifies the LR-TDDFT equation by reducing it to a standard Hermitian eigenproblem,

$$
\mathrm{AX}_{p}=\omega_{p} \mathrm{X}_{p} .
$$

We use the block Davidson method ${ }^{58}$ to solve this eigenproblem, as the matrix A itself is a diagonally dominant one and only few excited states are typically in interest.

A significant advantage of the Davidson algorithm follows from its iterative nature. The algorithm approximates target eigenvectors by iteratively refining a set of trial vectors $\mathrm{X}_{p}-$ one vector for each excited state $p$ in question-and only the action of the response operator on these vectors $\mathrm{AX}_{p}$ needs to be known. In our implementation, we use trial vectors in form of contracted response orbitals $\phi_{i ; p \sigma}^{(1)}(\mathbf{r})$, which is a linear combination of virtual Kohn-Sham orbitals $\phi_{a ; \sigma}(\mathbf{r})$,

$$
\phi_{i ; p \sigma}^{(1)}(\mathbf{r})=\sum_{a}^{\text {LUMO }} \mathrm{X}_{i a ; p \sigma} \phi_{a ; \sigma}(\mathbf{r}),
$$

obtained from solving the ground-state KS-DFT equations. The squared contraction coefficients $\left|\mathrm{X}_{i a ; p \sigma}\right|^{2}$ thus can be thought as a probability of an electron transition between the $i$ th occupied and ath virtual KS orbitals. 
By analogy with occupied ground-state KS orbitals $\phi_{i, \sigma}$, the contracted response orbitals are expanded as a linear combination of atomic basis functions $\{\chi\}$,

$$
\phi_{i ; \sigma}(\mathbf{r})=\sum_{\mu=1}^{\mathrm{N}} \mathrm{C}_{\mu i ; \sigma}^{(0)} \chi_{\mu}(\mathbf{r}), \quad \phi_{i ; p \sigma}^{(1)}(\mathbf{r})=\sum_{\mu=1}^{\mathrm{N}} \mathrm{C}_{\mu i ; p \sigma}^{(1)} \chi_{\mu}(\mathbf{r}),
$$

subject to orthogonality conditions,

$$
\begin{aligned}
& \operatorname{Tr}\left(C_{\sigma}^{(0), T} S_{p \sigma}^{(1)}\right)=0, \\
& \operatorname{Tr}\left(C_{p \uparrow}^{(1), T} S C_{q \uparrow}^{(1)}\right)+\operatorname{Tr}\left(C_{p \downarrow}^{(1), T} S C_{q \downarrow}^{(1)}\right)=\delta_{p q},
\end{aligned}
$$

where $S_{\mu v}=\left\langle\chi_{\mu} \mid \chi_{\nu}\right\rangle$ is the overlap matrix and $\delta_{p q}$ is the Kronecker delta. The calculation of the action of the response operator on trial vectors, expressed in the atomic basis set, is then performed in the following steps, which are essentially the same steps as required for building the Kohn-Sham matrix in ground-state DFT using the Gaussian and plane wave method: 28

1. For every spin component $\sigma$, construct the response density matrix,

$$
P_{p \sigma}^{(1)}=\frac{1}{2}\left(C_{\sigma}^{(0)} C_{p \sigma}^{(1), T}+C_{p \sigma}^{(1)} C_{\sigma}^{(0), T}\right) .
$$

The initial guess is formed from energetically ordered single orbital excitations. Note that the response density matrix is not a pure one, as it does not fulfil the idempotent property,

$$
\mathrm{P}_{p \sigma}^{(1)} \mathrm{SP}_{p \sigma}^{(1)} \mathrm{S} \neq \mathrm{P}_{p \sigma}^{(1)} \mathrm{S}
$$

Map the response density onto a real-space grid $\mathrm{P}_{p \sigma}^{(1)} \rightarrow \rho_{p \sigma}^{(1)}(\mathbf{r})$ and the corresponding reciprocal-space grid by performing the fast Fourier transformation (FFT),

$$
\rho_{p \sigma}^{(1)}(\mathbf{G})=\operatorname{FFT}\left[\rho_{p \sigma}^{(1)}(\mathbf{r})\right] .
$$

2. Compute the energy difference term,

$$
\mathrm{A}^{\mathrm{E}} \mathrm{C}_{p \sigma}^{(1)}=\mathrm{F}_{\sigma} \mathrm{C}_{p \sigma}^{(1)}-\epsilon_{\sigma} \mathrm{SC}_{p \sigma}^{(1)}
$$

where $F_{\sigma}$ and $S$ are Kohn-Sham and overlap matrices, respectively, and $\epsilon_{\sigma}$ is a diagonal matrix of Kohn-Sham orbital energies.

3. Compute the Coulomb term by

(a) solving the Poisson equation on the reciprocal grid, $\rho_{p \sigma}^{(1)}(\mathbf{G}) \rightarrow v_{p \sigma}^{(1)}(\mathbf{G})$

(b) calculating components of the electrostatic potential on the real-space grid using the inverse FFT, $v_{p \sigma}^{(1)}(\mathbf{r})$ $=\mathrm{FFT}^{-1}\left[v_{p \sigma}^{(1)}(\mathbf{G})\right]$.

4. Compute the adiabatic XC term by evaluating the integral,

$$
\bar{f}_{\mathrm{xc} ; p \sigma}(\mathbf{r})=\sum_{\tau=\uparrow, \downarrow} \eta_{\tau} \int f_{\mathrm{xc} ; \sigma \tau}\left(\mathbf{r}, \mathbf{r}^{\prime}\right) \rho_{p \tau}^{(1)}\left(\mathbf{r}^{\prime}\right) \mathrm{d} \mathbf{r}^{\prime},
$$

on the real-space grid. The scaling factors $\left(\eta_{\uparrow}, \eta_{\downarrow}\right)$ are equal to $(2,0)$ or $(1,-1)$ for singlet and triplet states computed using spin-unpolarised electron density, or $(1,1)$ otherwise.
5. Transform the sum of electrostatic potential and the XC term from the grid representation into a matrix representation in the atomic basis set by evaluating expectation values,

$$
\left(\mathrm{A}^{\mathrm{J}} \mathrm{C}_{p \sigma}^{(1)}+\mathrm{A}^{\mathrm{XC}} \mathrm{C}_{p \sigma}^{(1)}\right)_{\mu \nu}=\int \chi_{\mu}^{*}(\mathbf{r})\left(v_{p \sigma}^{(1)}(\mathbf{r})+\bar{f}_{\mathrm{xc} ; p \sigma}(\mathbf{r})\right) \chi_{v}(\mathbf{r}) \mathrm{d} \mathbf{r} .
$$

6. Using precomputed ERIs over atomic basis functions $(\mu \nu \mid \lambda \xi)$, compute matrix elements of the exact-exchange operator,

$$
\left(K_{p \sigma}\right)_{\mu \lambda}=\sum_{\nu \xi}(\mu \nu \mid \lambda \xi)\left(P_{p \sigma}^{(1)}\right)_{\nu \xi} .
$$

The exact-exchange action term is then calculated as a matrix product scaled by the amount of the exact exchange,

$$
c_{\mathrm{HFX}} \mathrm{A}^{\mathrm{HFX}} \mathrm{C}_{p \sigma}^{(1)}=\mathrm{c}_{\mathrm{HFX}} \mathrm{K}_{p \sigma} \mathrm{C}_{\sigma}^{(0)} .
$$

7. When combined with ADMM (see Sec. II B), instead of the previous step, compute the matrix elements of the exactexchange operator in an auxiliary basis set $\{\tilde{\chi}\}$ using the auxiliary density matrix P from Eq. (4),

$$
\left(\tilde{\mathrm{K}}_{p \sigma}\right)_{\tilde{\mu} \tilde{\lambda}}=\sum_{\tilde{v} \tilde{\xi}}(\tilde{\mu} \tilde{v} \mid \tilde{\lambda} \tilde{\xi})\left(\tilde{\mathrm{P}}_{p \sigma}^{(1)}\right)_{\tilde{\nu} \tilde{\xi}}
$$

and then project the obtained matrix back to the primary basis set,

$$
\mathrm{K}_{p \sigma}=\mathrm{O}^{\mathrm{T}} \tilde{\mathrm{K}}_{p \sigma} \mathrm{O}
$$

Repeating steps 4 and 5 we also compute two compensation XC terms using a reference (semi-)local XCfunctional in accordance with Eq. (8).

8. All components of the action matrix for the given response wave function (steps 2, 5, and 6) are then summed up. Once obtained for all excited states in question, these action matrices are then used in the block Davidson algorithm ${ }^{58}$ to compute residuals and to refine the response wave functions.

With no periodic boundary conditions, having the optimised response wave function for the $p$ th transition $\Psi_{p}$, one can compute the associated oscillator strength using the classic expression for dipole integrals in the "length" form, ${ }^{54}$

$$
f_{p}=\frac{2}{3} \omega_{p} \sum_{q=x, y, z}\left|\left\langle\Psi_{p}|q| \Psi_{0}\right\rangle\right|^{2} .
$$

The above expression can be recast in terms of contracted expansion coefficients,

$$
f_{p}=\frac{2}{3} \omega_{p} \sum_{q=x, y, z}\left|\operatorname{Tr}\left[C_{p}^{(1), \mathrm{T}} \mathrm{SC}^{\mathrm{virt} .} \mathrm{C}^{\mathrm{virt} . \mathrm{T}} \mathrm{Q}_{q} \mathrm{C}^{(0)}\right]\right|^{2},
$$

where the matrix elements of the dipole operator in atomic basis set are

$$
\mathrm{Q}_{q, \mu \nu}=\left\langle\chi_{\mu}|q| \chi_{v}\right\rangle
$$

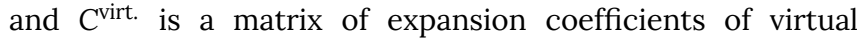
Kohn-Sham orbitals $\phi_{a ; \sigma}(\mathbf{r})$ in the atomic basis set. Using the 
commutation relation between Hamiltonian and position operators

$$
[\hat{\mathrm{H}}, \hat{\mathbf{r}}]=-\nabla
$$

we can rewrite Eq. (A21) in the equivalent "velocity" form,

$f_{p}=\frac{2}{3} \omega_{p} \sum_{q=x, y, z}\left|\operatorname{Tr}\left[C_{p}^{(1), T} S C^{\text {virt. }}\left\{\mathrm{W} \otimes\left(C^{\text {virt.,T }} \frac{\partial S}{\partial q} C^{(0)}\right)\right\}\right]\right|^{2}$,

to make it suitable for periodic boundary conditions. Here W is the inverse energy difference matrix between all virtual $(a)$ and occupied (i) Kohn-Sham orbitals,

$$
\mathrm{W}_{a i}=\left(\epsilon_{a}-\epsilon_{i}\right)^{-1} \text {. }
$$

$\frac{\partial S}{\partial q}$ is a matrix containing the first partial derivative of overlap integrals along the qth direction,

$$
\left(\frac{\partial \mathrm{S}}{\partial q}\right)_{\mu \nu}=\left\langle\chi_{\mu} \mid \frac{\partial \chi_{v}}{\partial q}\right\rangle
$$

and the symbol $\otimes$ denotes the element-wise (Hadamard) product.

\section{REFERENCES}

${ }^{1}$ A. I. Popov, E. A. Kotomin, and J. Maier, Nucl. Instrum. Methods Phys. Res., Sect. B 268, 3084 (2010).

${ }^{2}$ G. Pacchioni, Solid State Sci. 2, 161 (2000).

${ }^{3}$ A. L. Shluger, A. S. Foster, J. L. Gavartin, and P. V. Sushko, in Nano and Giga Challenges in Microelectronics, edited by J. Greer, A. Korkin, and J. Labanowski (Elsevier, 2003), pp. 151-222.

${ }^{4}$ M. V. Ganduglia-Pirovano, A. Hofmann, and J. Sauer, Surf. Sci. Rep. 62, 219 (2007).

${ }^{5}$ Y. Chen, R. T. Williams, and W. A. Sibley, Phys. Rev. 182, 960 (1969).

${ }^{6}$ R. C. Whited and W. C. Walker, Phys. Rev. Lett. 22, 1428 (1969).

${ }^{7}$ L. A. Kappers, R. L. Kroes, and E. B. Hensley, Phys. Rev. B 1, 4151 (1970).

${ }^{8}$ G. H. Rosenblatt, M. W. Rowe, G. P. Williams, Jr, R. T. Williams, and Y. Chen, Phys. Rev. B 39, 10309 (1989).

${ }^{9}$ A. Lushchik, T. Kärner, C. Lushchik, E. Vasil'chenko, S. Dolgov, V. Issahanyan, and P. Liblik, Phys. Status Solidi C 4, 1084 (2007).

${ }^{10}$ E. Feldbach, R. Jaaniso, M. Kodu, V. P. Denks, A. Kasikov, P. Liblik, A. Maaroos, H. Mändar, and M. Kirm, J. Mater. Sci.: Mater. Electron. 20, 321 (2009).

${ }^{11}$ F. Illas and G. Pacchioni, J. Chem. Phys. 108, 7835 (1998).

${ }^{12}$ C. Sousa and F. Illas, J. Chem. Phys. 115, 1435 (2001).

${ }^{13}$ M. A. Monge, R. Gonzalez, J. E. M. Santiuste, R. Pareja, Y. Chen, E. A. Kotomin, and A. I. Popov, Nucl. Instrum. Methods Phys. Res., Sect. B 166, 220 (2000).

${ }^{14}$ R. González, M. A. Monge, J. E. M. Santiuste, R. Pareja, Y. Chen, E. Kotomin, M. M. Kukla, and A. I. Popov, Phys. Rev. B 59, 4786 (1999).

${ }^{15}$ P. Rinke, A. Schleife, E. Kioupakis, A. Janotti, C. Rödl, F. Bechstedt, M. Scheffler, and C. G. Van de Walle, Phys. Rev. Lett. 108, 126404 (2012).

${ }^{16}$ G. P. Summers, T. M. Wilson, B. T. Jeffries, H. T. Tohver, Y. Chen, and M. M. Abraham, Phys. Rev. B 27, 1283 (1983).

${ }^{17}$ T. M. Wilson and R. F. Wood, J. Phys. Colloq. 37, C7 (1976).

${ }^{18}$ C. H. Lien, Y. S. Chen, H. Y. Lee, P. S. Chen, F. T. Chen, and M. -J. Tsai "The highly scalable and reliable hafnium oxide ReRAM and its future challenges," 2010 10th IEEE International Conference on Solid-State and Integrated Circuit Technology, Shanghai, China, 2010 (IEEE, 2010), pp. 1084-1087.

${ }^{19}$ H. Akinaga and H. Shima, Proc. IEEE 98, 2237 (2010).

${ }^{20}$ B. Traoré, P. Blaise, E. Vianello, E. Jalaguier, G. Molas, J. F. Nodin, L. Perniola, B. De Salvo, and Y. Nishi, in 2014 IEEE International Reliability Physics Symposium (IEEE, 2014) p. 5E.2.
${ }^{21}$ R. Degraeve, A. Fantini, G. Gorine, P. Roussel, S. Clima, C. Y. Chen, B. Govoreanu, L. Goux, D. Linten, M. Jurczak et al., in 2016 IEEE International Reliability Physics Symposium (IEEE, 2016) p. 6C.1.

${ }^{22}$ R. Öttking, S. Kupke, E. Nadimi, R. Leitsmann, F. Lazarevic, P. Plänitz, G. Roll, S. Slesazeck, M. Trentzsch, and T. Mikolajick, Phys. Status Solidi A 212, 547 (2015).

${ }^{23}$ S. R. Bradley, G. Bersuker, and A. L. Shluger, J. Phys.: Condens. Matter 27, 415401 (2015).

${ }^{24}$ T. V. Perevalov, V. S. Aliev, V. A. Gritsenko, A. A. Saraev, V. V. Kaichev, E. V. Ivanova, and M. V. Zamoryanskaya, Appl. Phys. Lett. 104, 071904 (2014).

${ }^{25}$ V. A. Gritsenko, T. V. Perevalov, and D. R. Islamov, Phys. Rep. 613, 1 (2016).

${ }^{26}$ D. M. Ramo, J. L. Gavartin, A. L. Shluger, and G. Bersuker, Phys. Rev. B 75, 205336 (2007)

${ }^{27}$ V. A. Gritsenko, D. R. Islamov, T. V. Perevalov, V. S. Aliev, A. P. Yelisseyev, E. E. Lomonova, V. A. Pustovarov, and A. Chin, J. Phys. Chem. C 120, 19980 (2016).

${ }^{28}$ J. VandeVondele, M. Krack, F. Mohamed, M. Parrinello, T. Chassaing, and J. Hutter, Comput. Phys. Commun. 167, 103 (2005).

${ }^{29}$ M. Guidon, J. Hutter, and J. VandeVondele, J. Chem. Theory Comput. 5, 3010 (2009).

${ }^{30} \mathrm{C}$. Adamo and V. Barone, J. Chem. Phys. 110, 6158 (1999).

${ }^{31}$ M. Ernzerhof and J. P. Perdew, J. Chem. Phys. 109, 3313 (1998).

${ }^{32}$ S. Goedecker, M. Teter, and J. Hutter, Phys. Rev. B 54, 1703 (1996).

${ }^{33}$ J. VandeVondele and J. Hutter, J. Chem. Phys. 127, 114105 (2007).

${ }^{34} \mathrm{M}$. Guidon, J. Hutter, and J. VandeVondele, J. Chem. Theory Comput. 6, 2348 (2010).

${ }^{35}$ P. Merlot, R. Izsák, A. Borgoo, T. Kjærgaard, T. Helgaker, and S. Reine, J. Chem. Phys. 141, 094104 (2014).

${ }^{36}$ A. F. Izmaylov and G. E. Scuseria, J. Chem. Phys. 129, 034101 (2008).

${ }^{37}$ R. M. Hazen, Am. Mineral. 61, 266 (1976), see http:/ / www.minsocam.org/ ammin/AM61/AM61_266.pdf.

${ }^{38}$ R. E. Hann, P. R. Suitch, and J. L. Pentecost, J. Am. Ceram. Soc. 68, C285 (1985).

${ }^{39}$ M. Balog, M. Schieber, M. Michman, and S. Patai, Thin Solid Films 41, 247 (1977).

${ }^{40}$ L. E. Halliburton, D. L. Cowan, and L. V. Holroyd, Phys. Rev. B 12, 3408 (1975).

${ }^{41}$ C. J. Krap, M. Glasbeek, and J. D. W. Van Voorst, Phys. Rev. B 17, 61 (1978).

${ }^{42}$ A. Lushchik, C. Lushchik, K. Schwartz, F. Savikhin, E. Shablonin, A. Shugai, and E. Vasilchenko, Nucl. Instrum. Methods Phys. Res., Sect. B 277, 40 (2012).

${ }^{43}$ T. Koyama and T. Suemoto, Rep. Prog. Phys. 74, 076502 (2011).

${ }^{44}$ R. O. Jones and O. Gunnarsson, Rev. Mod. Phys. 61, 689 (1989).

${ }^{45}$ T. Timusk and W. Martienssen, Phys. Rev. 128, 1656 (1962).

${ }^{46}$ A. L. Shluger and K. Tanimura, Phys. Rev. B 61, 5392 (2000)

47J. Aarik, H. Mändar, M. Kirm, and L. Pung, Thin Solid Films 466, 41 (2004).

${ }^{48}$ K. P. McKenna, M. J. Wolf, A. L. Shluger, S. Lany, and A. Zunger, Phys. Rev. Lett. 108, 116403 (2012).

${ }^{49}$ D. M. Ramo, P. V. Sushko, and A. L. Shluger, Phys. Rev. B 85, 024120 (2012).

${ }^{50}$ D. M. Ramo, A. L. Shluger, J. L. Gavartin, and G. Bersuker, Phys. Rev. Lett, 99, 155504 (2007).

${ }^{51}$ A. T. Vink, J. Lumin. 9, 159 (1974).

${ }^{52}$ A. Shluger, E. Kotomin, and L. Kantorovich, Solid State Commun. 42, 749 (1982).

${ }^{53}$ E. V. Ivanova, M. V. Zamoryanskaya, V. A. Pustovarov, V. S. Aliev, V. A. Gritsenko, and A. P. Yelisseyev, J. Exp. Theor. Phys. 120, 710 (2015).

${ }^{54}$ M. E. Casida, J. Mol. Struct.: THEOCHEM 914, 3 (2009).

${ }^{55}$ A. Dreuw, J. L. Weisman, and M. Head-Gordon, J. Chem. Phys. 119, 2943 (2003).

${ }^{56}$ M. Iannuzzi, T. Chassaing, T. Wallman, and J. Hutter, Chimia Int. J. Chem. 59, 499 (2005).

${ }^{57}$ S. Hirata and M. Head-Gordon, Chem. Phys. Lett. 314, 291 (1999)

${ }^{58}$ M. Crouzeix, B. Philippe, and M. Sadkane, SIAM J. Sci. Comput. 15, 62 (1994).

${ }^{59}$ T. Kowalczyk, S. R. Yost, and T. V. Voorhis, J. Chem. Phys. 134, 054128 (2011). 